\title{
Interferometric Measurement of Heat Transfer above New Generation Foam Concrete
}

\author{
Elena Pivarčiová ${ }^{1}$, Pavol Božek ${ }^{2}$, Kséniia Domnina $^{3}$, Emil Škultéty $^{1}$, Sergey Fedosov $^{4}$ \\ ${ }^{I}$ Department of Manufacturing and Automation Technology, Faculty of Environmental and Manufacturing Technology, \\ Technical University in Zvolen, Študentská 26, 96053 Zvolen, Slovakia, pivarciova@tuzvo.sk, emco313@azet.sk \\ ${ }^{2}$ Institute of Production Technologies, Faculty of Materials Science and Technology, \\ Slovak University of Technology, J. Bottu 25, 91724 Trnava, Slovakia, pavol.bozek@stuba.sk \\ ${ }^{3}$ Votkinsk branch of Izhevsk State Technical University, Shuvalova Street 1, 427430 Votkinsk, Udmurt Republic, Russia, \\ kseniya_domnina@bk.ru \\ ${ }^{4}$ Ivanovo State Polythechnic University, Sheremetevskiy prospect 21, 153000 Ivanovo, Ivanovo region, Russia, fedosov- \\ academic53@mail.ru
}

\begin{abstract}
The contribution is focused on investigating the heat transfer via natural convection which originated as an effect of changed air density by heating the horizontal sample in the area given. For this research we used samples of a new material made in the Russian Federation - the foam concrete which was reinforced by PET fibres. The samples were heated by an electric heating device from the bottom. The temperature fields originating above the horizontal sample surface were visualised by means of the holographic interferometric contactless method in real time. The holographic interferograms of the temperature field were analysed, and then the local heat transfer parameters were calculated: the heat transfer coefficient $\alpha$, and the heat conductivity coefficient $\lambda$.
\end{abstract}

Keywords: Holographic interferometry, Mach-Zehnder interferometer, heat transfer, foam concrete.

\section{INTRODUCTION}

Nowadays, regarding the very specific requirements for heat protection of buildings and structures, the demand for high-quality structural heat-insulating porous materials, including non-autoclaved foam concrete, has significantly increased. Foam concrete has found wide application in industrial and civil low-rise construction due to the fact that compared with ordinary concrete it has a number of advantages, such as the best thermal insulation properties, high processability, environmental friendliness, and water resistance. It is used for external and internal masonry walls, insulation of roofs and attics, sound, and thermal insulation of interfloor overlappings, and thermal insulation of pipelines.

On the other hand, the promising direction of foam concrete production using non-autoclave technology has a number of drawbacks as well, and as such it needs quality improvement in terms of the finished product. The solution to several technological issues en bloc could be represented by a dispersed reinforcement of foam concrete with various types of fibres.

Studies of a number of authors [1] confirm that the introduction of fibres in the foam concrete mixture provides:
- improving the strength characteristics of concrete by increasing the crack, impact, and wear resistance, static strength under various force effects [2];

- improving the operational reliability of structures under the influence of aggressive environment by improving the structure of the material [3];

- reducing the shrinkage deformations through the formation of a spatial reinforcing frame [4].

A very promising, however, less known material used also for concrete reinforcement are polyethylene terephthalate (PET) fibres obtained via their waste recycling. PET fibre is a synthetic thermoplastic polymer which can improve the concrete quality.

However, the usage of such fibre types for concrete reinforcement has been considered not economical, particularly due to its high costs. The good news is that the PET-raw material conversion laboratory based in the Votkinsk Branch of Izhevsk State Technical University has the platform for such fibre production. The laboratory staff has developed a new method of PET-fibre acquisition, reducing thus the production costs, and at the same time solving a number of environmental issues regarding its application. 
At this stage, the composition of structural heat that is insulating the non-autoclaved foam concrete reinforced by PET fibre is being developed and patented; the influence of polyethylene terephthalate fibre on the structure, as well as its properties, are being established. In addition, it has been proven that the PET fibre integration in the amount of $0.7 \%$ of the total mass into the foam concrete mixture composition has led to the improvement of the strength properties by $40 \%$ [5]. Here it has to be complemented, that the heat-insulating properties of the foam concrete reinforced by PET fibre have not been studied so far.

Experimental methods play an important role in solving the tasks within the base or applied research. The methods are used to verify the results of theoretical calculations or precision of mathematical calculations, and sometimes, they represent the only solution. The optical method of holographic interferometry belongs also to these experimental methods.

The holographic interferometry provides its user with the information acquisition about the format of an observable (macroscopic) interferometric figure in which there is encoded information characterising at least one physical state of the object observed [6].

A few researchers have dealt with the research of heat transfer by means of holographic interferometry, e.g. Mayinger, Panknin [7] dealt with the holography in heat and mass transfer. Taucher [8] carried out several experiments in heat and mass transfer by means of holographic interferometry. Binu et al. [9] described the whole field NDT of porous materials using digital holography. Černecky, Pivarčiova [6] observed temperature fields by holographic interferometry. Pavelek et al. [10] described the visualisation and optical measurement methods in detail. Herman [11] executed a theoretical analysis of the quantitative visualisation of heat transfer in oscillatory and pulsatile flows. Lédl et al. [12] used holographic interferometry for the measurement of the temperature field in a fluid. Haridas, Sobhan [13] measured convective heat transfer from a vertical flat plate using the Mach-Zehnder interferometer with the wedge fringe setting. Dančová et al. [14] described holographic interferometry as a tool for visualisation of temperature fields in the air. Yogesh et al. [15] provided a review on natural convection heat transfer through inclined parallel plates. Černecky et al. [16] dealt with the heat transfer area roughness on heat transfer enhancement by a forced convection. Verma et al. [17] wrote about optical interferometers: their principles and applications in transport phenomena. Zhu et al. [18] described the temperature measurement of an inclined cylinder using optical interferometry. And finally, Anooplal, Binoy [19] focused on the investigation of the heat transfer properties of nano-fluids using Michelson interferometry.

In the contribution we focus on the observation and analysis of temperature fields through the new material - foam concrete reinforced by PET fibres, which has been developed in the Russian Federation. We utilized the method of holographic interferometry allowing us to visualize the temperature fields without interrupting the area observed, and at the same time, it has allowed making the transparent inhomogeneities in the whole observed area visible. This provided us with a compact image of the researched area in real time.

The method uses the change of air density which causes the change of refractive index and influences thus the spread of light beams. The method of interferometry allows measuring the refractive index while observing the temperature fields at the same time.

Considering the quantitative analysis of holographic interferogram figures, the temperatures and local heat transfer coefficients - the heat transfer coefficient $\alpha$ and the thermal conductivity coefficient $\lambda$ - were calculated.

\section{SUBJECT \& METHODS}

\section{A. Foam concrete}

The structural heat efficiency of the insulating materials is estimated by the thermal conductivity coefficient $\lambda$. Depending on the density of the reinforced foam concrete, the thermal conductivity coefficient ranges from 0.07 up to $0.35 \mathrm{~W} /\left(\mathrm{m}^{\circ} \mathrm{C}\right)$. The higher the density, the higher the thermal conductivity coefficient. The heat conductivity of foam concrete also depends on the nature of its porosity, the size, as well as on the number of pores, the content of water vapour, or water in a drip state, and on the humidity.

The structural heat samples of the insulating non-autoclave foam concrete with the density of $600 \mathrm{~kg} / \mathrm{m}^{3}$ made in the Votkinsk Branch of Izhevsk State Technical University via the classical production technology were used for experimental research. PC400 Portland cement, river sand, water, a foaming agent, and reinforcing PET fibre were used as raw materials.

\section{B. Method of holographic interferometry for transparent object research}

Holographic-interference methods for researching the transparent objects are based on mutual activity of two coherent waves, one of which is deformed by the transfer through the optical inhomogeneity, and the other one transfers through the reference unimpaired area. Subsequently, on the focusing screen, it is possible to observe alternating maxima and minima of light intensity (interference stripes), and hence determine the changes of the refraction index in the researched area. The holographic interferograms allow the direct and qualitative evaluating of the action observed. For the qualitative evaluation it is essential to determine the arrangement of the refraction index, and subsequently, calculate the required physical quantities [20].

In the experiment we used a real-time method "InfiniteFringe". Sometimes, the method is also called "living fringes". The real-time method allows observing of interference figure changes and simultaneously also observing the changes of phase inhomogeneity in real time. 
On the holographic board the reference state is recorded (usually by the temperature of the surroundings) - the image of an object wave $\varepsilon$ (without the phase inhomogeneity), and the image of a reference wave $\varepsilon_{\mathrm{r}}$ (Fig.1.a)).

Once the hologram is photochemically processed, it is located in the same place and in the optical arrangement it was exposed.

In the beginning (i.e. before heating), the object wave is identical with the original state for which the reference hologram was recorded. By the adjustment of the interferometer of the infinite stripe width no interference stripes will be observed (Fig.1.b)).

Now, it is possible to start with the heat transfer we would like to research. Heating causes the refractive index. If there is a load of heat, the object wave will be shifted by a phase due to the change in temperature (Fig.1.c)).

The difference between the reference state recorded earlier and the new state in the measured area, i.e. the phase shift between reference and object volumes is visualized in the form of a macroscopic interference pattern. The observer sees the interference stripes originating by the interference of both waves moving due to the change in temperature. The interference pattern can be observed and recorded in real time.
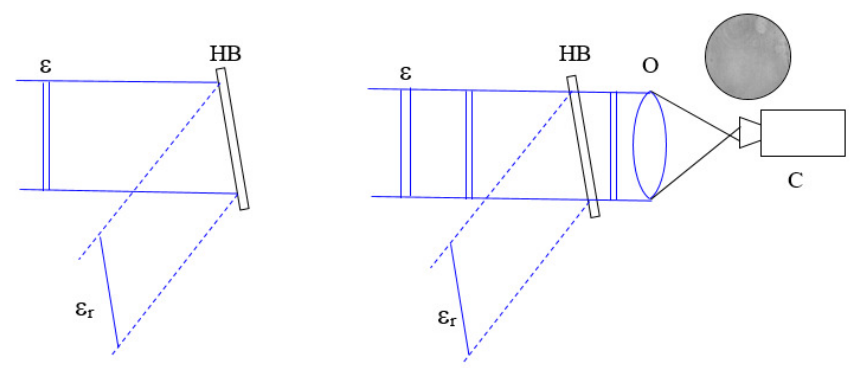

a)

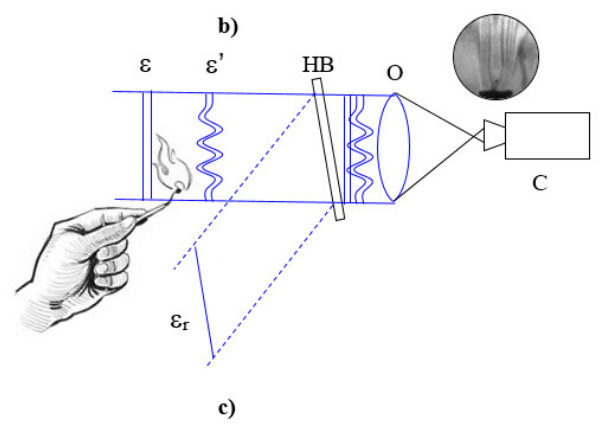

Fig.1. Real time method. a) record of object and reference waves on a holographic board, b) reconstruction without optical inhomogeneity, c) reconstruction by optical inhomogeneity activity, $\varepsilon$ - object light wave, $\varepsilon^{\prime}$ - deformed object light wave after temperature field transfer, $\varepsilon_{\mathrm{r}}-$ reference light wave, $\mathrm{HB}$ - holographic board, $\mathrm{O}$ - object lens, $\mathrm{C}$ - camera.

The advantage of the real time record is represented by the possibility to observe the time course of the interference image development via one hologram in the beginning state as well as by the possibility to record the stages of the object state, and what is more, it is also possible to observe the dynamic actions.

\section{Mach-Zehnder interferometer}

The holographic Mach-Zehnder interferometer for researching transparent objects in real time belongs to the mostly used interferometers for visualizing the 2D transparent objects, e.g. the temperature fields. A typical optical set for the holographic interferometry is shown in Fig.2. This interferometer type allows only studying of 2D or rotary symmetric objects, therefore, it is primarily utilized for the research of temperature fields in cracks or in the surroundings of simple rotary symmetric forms.

The holographic Mach-Zehnder interferometer can interfere with a holographically recorded object volume corresponding with the reference object state (reconstructed via a holographic reference volume), and a real object volume whose wave surface was deformed by transferring through the measured object. By setting the interferometer to the infinite stripe width, the geometry routes of the object and reference lines have to be of the same length. In this case, the stripes originate only due to the changes in the refraction index.

After the reflection from the mirror, the laser beam $\left(\mathrm{M}_{1}\right)$ is divided on the division board (D) into two volumes - object and reference volumes. A part of the beam - divider (D) transmits and the light is spread in the original direction. By the means of further mirrors $\left(\mathrm{M}_{2}, \mathrm{M}_{3}\right)$, and by the mirror located in the cardan suspension $(\mathrm{CM})$, the beams are directed into the place where the holographic board (HB) is located. To be able to record a wider field, the beams have to be widened via telescopic sets $\left(\mathrm{MO}_{1}+\mathrm{C}_{1}+\mathrm{O}_{1}, \mathrm{MO}_{2}+\mathrm{C}_{2}+\right.$ $\mathrm{O}_{2}$ ) to wide volumes.

The object volume of beams (OL) transferring the phase object above the testing solid (TO) strikes upon the holographic board (HB) gripped in a holder (DH). The other volume of parallel beams (reference line RL) strikes upon the mirror placed in the cardan suspension.

By turning the mirror (CM) around two mutually independent axes, it is possible to adjust the finite or infinite stripe width in the reference area behind the hologram.

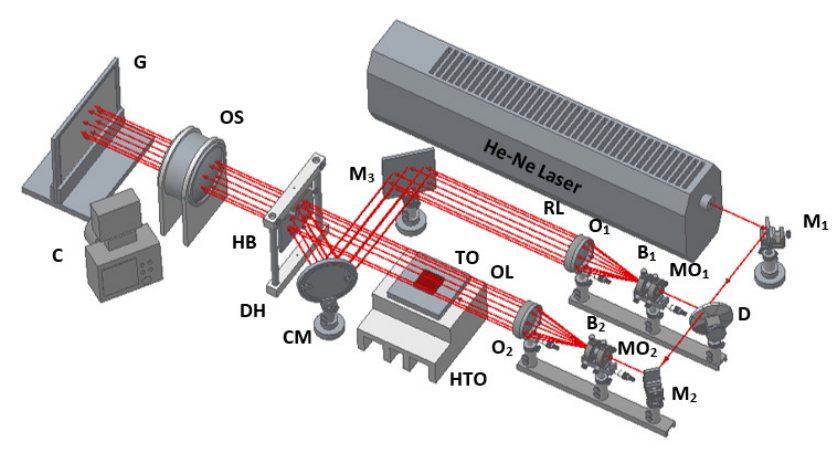

Fig.2. Scheme of the holographic variable - one-wave MachZehnder interferometer. OL - object line, RL - reference line, D divider, HB - holographic board, $\mathrm{DH}$ - holder of holographic board, $\mathrm{CM}-$ mirror in cardan suspension, $\mathrm{O}_{1}, \mathrm{O}_{2}-$ object lenses, $\mathrm{M}_{1}, \mathrm{M}_{2}$, $\mathrm{M}_{3}$ - mirrors, $\mathrm{B}_{1}, \mathrm{~B}_{2}-$ perforated blinds, $\mathrm{MO}_{1}, \mathrm{MO}_{2}-$ microscopic object lenses, TO - testing object, HTO - holder for gripping the testing object, $\mathrm{C}$ - camera, $\mathrm{G}$ - ground-glass, OS - object lenses system. 
The hologram is obtained by the simultaneous exposure of the holographic board via two light volumes by the homogeneous arrangement of the refraction index in the measured area, i.e. without entering the phase object.

Once the holographic board is processed photochemically, it is possible to reconstruct the original object volume which in real time interferes with the real object volume deformed by optical inhomogeneities, i.e. if it comes to an interruption in any place in which the light refraction index changes, e.g. if the air density is changed due to the changes in temperatures, the local values of the optical light route change as well. Any change in the arrangement of the refraction index deforms the real object wave and appears in the form of interference stripes occurrence.

As the source for the holographic interferometry we used He-Ne laser with the wave length of $\lambda=0.6328 .10^{-6} \mathrm{~m}$ with the continuous power exposure of $50 \mathrm{~mW}$ and having the required time and space coherence.

\section{Analysis of holographic interferograms}

Regarding the holographic interferogram analysis, it is assumed that the experiment is mainly two-dimensional along the optical axis, therefore, the refractive index is constant along every light beam. Further on, an equation connecting the refractive index with the temperature (via its density) is needed so that the refractive index could be transferred to the absolute temperature [17].

For ideal gases whose refractive index differs only a little from 1, the Gladstone-Dale relation follows [6], [10]:

$$
\frac{n-1}{\rho}=K
$$

where $n$ - refractive index, $\rho$ - density, $K$ - Gladstone-Dale constant.

The Gladstone-Dale constant depends on the gas type and on the wave length of used light. The relation (1) is basically an equation of the state for an ideal gas since it binds two state quantities, i.e. the refractive index and gas density. The refractive index of dry air for the light wave length of $\lambda=632.8 \mathrm{~nm}$ (for He-Ne laser) can be calculated according to [10], and [6]:

$$
n=1+2.256 \cdot 10^{-4} \rho=1+7.8607 \cdot 10^{-7} \frac{p}{T}
$$

where $n$ - refractive index, $\rho$ - density, $p$ - air pressure, $T-$ air temperature.

The air humidity influences the refractive index only a little. To derive the air refractive index according to the temperature, the relation follows [6]:

$$
\frac{\partial n}{\partial T}=-7.8607 \cdot 10^{-7} \frac{p}{T^{2}},
$$

where $n$ - refractive index, $p-$ air pressure, $T$ - air temperature.
To derive the relations necessary for the quantitative evaluation of interferograms, we need to describe the interference origin in a mathematical way.

The planar coherent light wave transferring the transparent area with the length $l$ is deformed while the ideal interferometry assumes the deformation of the wave surface and neglects the light beam curvature which should always be perpendicular to the light wave front. In the evaluation of the interferograms, the equation for the optical routes difference $\Delta o$ caused by the change of the environment refractive index via an optical inhomogeneity is assumed:

$$
\Delta o(x, y)=\int_{0}^{l}\left[n(x, y)-n_{\infty}\right] \mathrm{d} z,
$$

where $\Delta o$ - optical routes difference, $l$ - length of model, $n(x$, $y)$ - refractive index in the points $x, y$ of phase object, $n_{\infty}-$ refractive index in the reference area (refractive index of measuring area without a phase object).

For a two-dimensional transparent object after the equation implementation (4), it follows:

$$
\Delta o(x, y)=l\left[n(x, y)-n_{\infty}\right] .
$$

If the deformed light wave interferes with the planar wave of the reference volume, an interferogram is originated. Then, to change the interferometry order $\Delta S(x, y)$, it follows:

$$
\Delta o(x, y)=\Delta S(x, y) . \lambda,
$$

where $\Delta S(x, y)$ - change of interferometry order, $\lambda$ - wave light length.

The interferometry research is aimed at calculating the physical quantities in observed transparent objects. The quantities can be derived from the primary quantities (light refractive index). If we consider a constant pressure, then the temperature arrangement can be calculated directly from the interference order.

The change of the interference order $\Delta S(x, y)$ gains the following values:

$\Delta S(x, y)=\ldots ;-2 ;-1 ; 0 ; 1 ; 2 ; \ldots$ in the positions of light stripes,

$\Delta S(x, y)=\ldots ;-1.5 ;-0.5 ; 0.5 ; 1.5 ; 2.5 ; \ldots$ in the positions of dark stripes.

The aforementioned way of interference order allocation follows for setting the interferometer onto an infinite width of interference stripes in the reference area (in the homogenous object area the change of the interference order $\Delta S(x, y)=0)$, when the reference and object waves in the homogenous object part are parallel.

From the equations (4) and (5) it is possible to derive the equation of an ideal interferometry of which the refractive index $n(x, y)$ in the evaluated area is stated as a function of the refractive index in the reference area $n_{\infty}$, and the changes of interference order $\Delta S(x, y)$, the light wave length $\lambda$, and the length of the model $l$ in the direction of beam spread are determined as well. 
The equation of an ideal interferometry is derived providing that the presented optical inhomogeneity causes a negligibly small deviation and shift of the light beam and has the shape according to [6].

$$
n(x, y)=n_{\infty}+\frac{\Delta S(x, y) \cdot \lambda}{l}
$$

where $n(x, y)$ - refractive index in $x, y$ point of the phase object, $n_{\infty}$ - refractive index in the reference area, $\Delta S(x, y)$ change of interference order, $\lambda$ - wave light length, $l$ - length of the model.

To evaluate the interferograms, it is necessary to know the physical regularity of the state quantities (density, temperature, and concentration) and the arrangement of the refractive index in the optical inhomogeneity caused by the change of the quantities mentioned.

The functional dependence of the temperature on the state quantities of the surroundings, on the length of the model, on the light wave length, and on the number of dark stripes from the position of the inhomogeneous area are determined according to [20]:

$$
T(x, y)=\frac{T_{\infty}}{1-0.805 \cdot \frac{T_{\infty}}{l \cdot p_{\infty}} \cdot\left(s-\frac{1}{2}\right)}
$$

where $T(x, y)$ - arrangement of temperatures, temperature in related interference order, $T_{\infty}$ - air temperature in reference area, $p_{\infty}$ - pressure in the given area, $s$-interference order, $\lambda$ - light wave length, $l$ - length of the model.

\section{E. Calculation of the heat transfer coefficient}

The heat transfer coefficient is a quantity used when we need to determine the share of heat between two areas separated by a fixed board-shaped counter made of a homogenous material. It can be calculated from an inverted value of the specific thermal resistance $R_{\mathrm{k}}$ of the heat transfer through the counter from one area to the other one. In civil engineering, the heat transfer coefficient is called the heat permeability and it is designated as $U$.

By the air circumfluence of the objects' surface with the temperature $T_{\infty}$ different from the surface temperature $T_{\mathrm{x}}$ in the position $x$, the local heat transfer between the surface and the air occurs here. The local coefficient value of the heat transfer $\alpha_{x}$ depends on many factors, e.g. on the type of the liquid, on the speed of the flow, on the shape of the surface, on the position of the observed area, or on the difference of temperatures between the surface and the air [20].

The local heat transfer coefficient value can be calculated from the equation [10]:

$$
\alpha_{x}=-\lambda_{v}\left(\frac{d T}{d y}\right)_{x} \frac{1}{T_{x}-T_{\infty}},
$$

where $\lambda$ - heat conductivity coefficient (determined by the surface temperature), $T_{\mathrm{x}}$ - surface temperature in the position $x, T_{\infty}$ - temperature of the surrounding area.

From the measured or calculated shape of the temperature profile, the difference of temperatures $T_{\mathrm{x}}-T_{\infty}$ and the temperature derivation can be expressed.

For calculating the local heat transfer coefficient, it follows [10]:

$$
\alpha_{x}=-\lambda \cdot \operatorname{tg}\left(\beta_{x}\right) \cdot \frac{1}{T_{x}-T_{\infty}} .
$$

This way of calculating the heat transfer coefficient is practically applied in the interferometric heat transfer research since the interferograms provide us with the detail temperature arrangement in the liquid [10].

\section{F. Calculation of heat conductivity coefficient (specific heat conductivity)}

We assume having a horizontal planar board heated from the bottom and with the surface temperature $t_{1}$ on the heated side and the temperature $t_{2}$ on the top. According to Fourier's law the density of the heat flow $q$ is:

$$
q=-\lambda \cdot \frac{\mathrm{d} T}{\mathrm{~d} x}
$$

where $\lambda$ is heat conductivity coefficient (specific heat conductivity).

For the density of stabilised heat transfer $q$ through the board with the width of $d$ it follows:

$$
q=\lambda \cdot \frac{T_{1}-T_{2}}{d}
$$

If the temperature of the area gas (or liquid) above the board is $t_{\infty}$, then according to Newton's law for the density of the heat flow it follows:

$$
q=\alpha \cdot\left(T_{2}-T_{\infty}\right)
$$

where $\alpha$ is the heat transfer coefficient.

From the equality of thermal heat flow density through the board it follows:

$$
\lambda \cdot \frac{T_{1}-T_{2}}{d}=\alpha \cdot\left(T_{2}-T_{\infty}\right),
$$

of which the unknown heat permeability coefficient of the board can be calculated:

$$
\lambda=\alpha \cdot d \cdot \frac{T_{2}-T_{\infty}}{T_{1}-T_{2}} .
$$




\section{RESULTS}

For heat transfer measurement above the sample the testing objects of foam concrete with the dimensions of $40 \times 40 \times 10 \mathrm{~mm}$ were used. Two electric board-shaped heaters with the overall input power of $300 \mathrm{~W}$ together with the possibility of manual regulation of the thermal capacity within $15-100 \%$ were used.

The holographic interferograms of the temperature field above the heated testing object were recorded by the surrounding area's temperature of $23.5^{\circ} \mathrm{C}(296.65 \mathrm{~K})$ and the pressure of $98100 \mathrm{~Pa}$.

The holograms were recorded by the setting of the MachZehnder interferometer onto infinite width of interference stripes in real time. During the experiments, we observed the temperature field arrangement above the testing object as it represents all three types of the heat transfer (conductivity, radiation, and convection) and the thermal-physical properties of the testing object as well.

The theory of heat sharing is quite expansive, it deals with the heat transfer processes. Heat transfer is an imbalanced thermo-dynamic activity running with a finite speed in space and time. The heat sharing is conditioned by the heat difference existence. The heat sharing is a very complex activity, nevertheless, it is possible to classify it into: heat sharing via conductivity, convection, and radiation. In real world these heat sharing types do not occur as isolated ones but in various combinations.

In our experiment, the heat transfer was a combined type of heat sharing between two areas separated by a counter. The heat transferred from the warmer area with the temperature $t_{1}$ and entered the fixed counter via convection, conductivity, and radiation, and it came out into the colder air with the temperature $t_{2}$ via convection, conductivity, and radiation again.

We focused on the solution to a simplified case: the heat transfer via natural convection caused by the change of air density in heating the horizontal sample in the area given. The difference in air densities caused by the different temperature on the surface of the heated sample and its surroundings, activated the buoyant forces causing thus the rise of the air; and at the same time the colder air flew into its place as the heat transfer via conductivity ran as the first one and the heat transfer via convection came only after. The higher the difference of the temperatures between the counter and the air, the more intensive the air convection was.

Figure of holographic interferograms obtained experimentally is shown in Fig.3.

The results of the interferometric visualisation of the temperature fields in transparent objects are represented by the images of interferograms which can be evaluated both by quantitative or qualitative ways. Regarding the fact that every line was the line of constant stage, it was also the line of the constant refractive index. When evaluating the interferograms of 2D temperature fields in a qualitative way, and by setting the interferometer onto the infinite width of stripes in the reference area, the interference stripes then represented the phase object spots with the same value of the refractive index, i.e. isotherms of the temperature fields (spots with a certain temperature). If they were close to each other, it meant there was a high thermal gradient (a large change of temperature in a small area). The stripes located further from each other presented areas with almost the same temperatures.
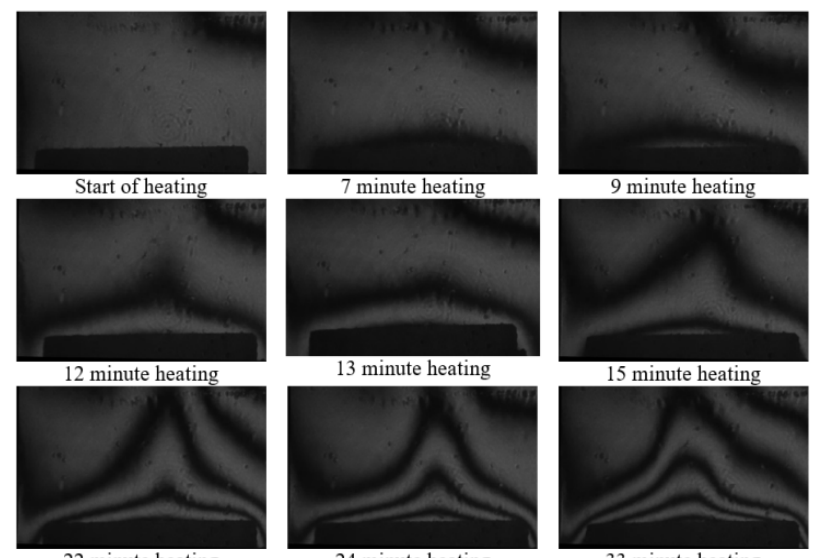

15 minute heating

22 minute heating

24 minute heating

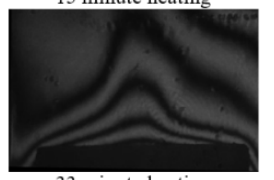

33 minute heating

Fig.3. Holographic interferograms of temperature fields.

The obtained records of the holographic interferograms usually provide us with the qualitative evaluation of the observed activity. The qualitative evaluation of interferogram images allows researching the shape of temperature fields, their mutual interactions, and their influence on local heat transfer parameters.

From the obtained holographic interferograms the quantitative analysis was carried out as well. For the quantitative evaluation of visualised records it was necessary to state the refractive index arrangement in the object, and subsequently calculate the arrangement of required physical quantities, in our case the temperatures were calculated according to (8). The determination of the refractive index change arrangement was possible via the interferogram of the recorded interference order change, as the interference order arrangement depended only on the refractive index arrangement in the observed area.

Fig.4. shows the course of temperatures above the heated sample after 33 minutes heating.

Further on, from the interferograms the local heat transfer coefficients $\alpha$ x were calculated according to (9) by using the temperature of the counter surface and the temperature and distance of the first interference stripe above the counter.

The images of the holographic interferograms showed here that where there was the lowest air flow, there was also the highest gradient, i.e. the temperature gradient was the highest one and the individual isotherms were close to each other.

In the areas where there was the air flow (by the finite width of the sample it was in the middle of the sample), the specific bell-shaped stripes occurred, and there the temperature gradient was the lowest one and the individual isotherms were more distant from each other.

The heat transfer coefficient alfa was evaluated in these areas of specific bell-shaped stripes. Fig.5. shows the calculated values of the local heat transfer after 33 minutes of heating on the spots of 20, 22, and $24 \mathrm{~mm}$ from the edge of the sample. 


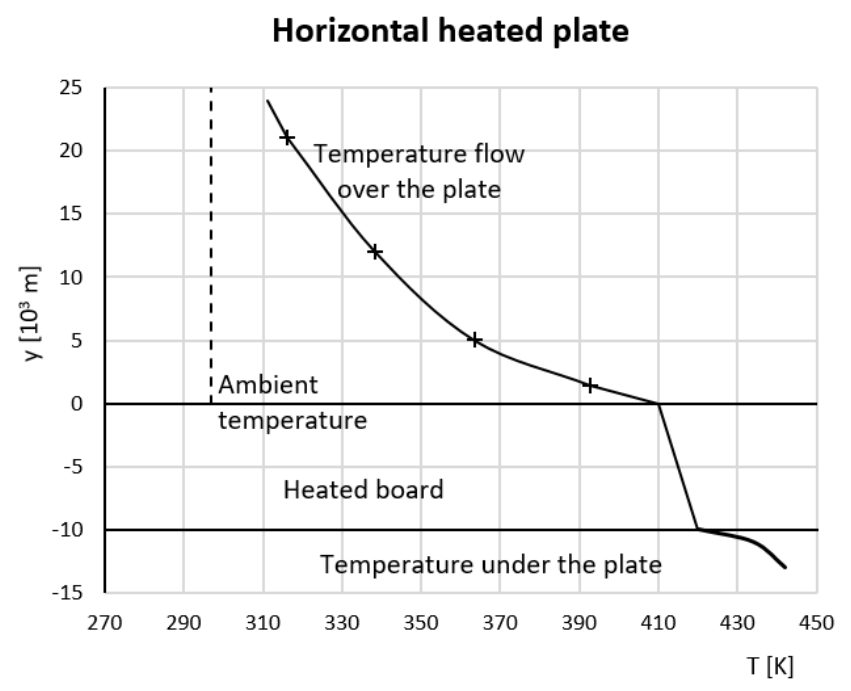

Fig.4. Course of temperatures above the heated sample after 33 minutes heating.
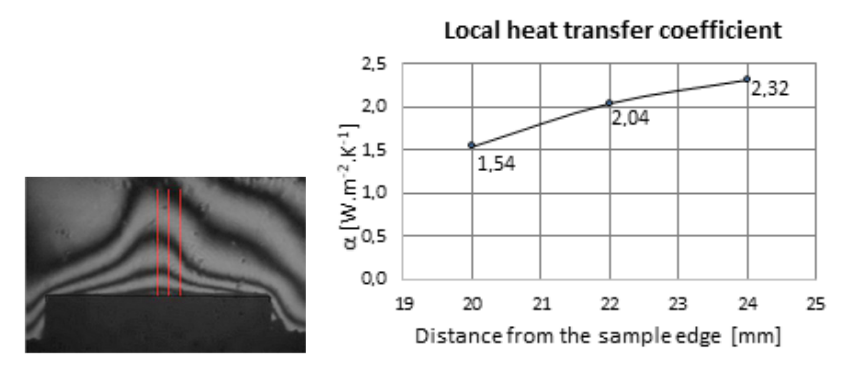

Fig.5. Local heat transfer coefficient.

The denser the stripes, the higher the heat transfer coefficient $\alpha$ was. In the specific bell-shaped interference stripes there was lower heat conductivity, and there was also higher resistance on larger distance, and lower gradient.

The higher the heat transfer coefficient, the higher the thermal flow transferring the area, and vice versa, the lower the heat transfer coefficient, the lower the thermal flow was as well, i.e. the heat resistance by entering the area was higher.

To calculate the heat conductivity coefficient, we used the local heat transfer coefficient $\alpha=1.54 \mathrm{~W} \cdot \mathrm{m}^{-2} \cdot \mathrm{K}^{-1}$ obtained by evaluating the cross-section of the interference stripes above the middle of the sample. By using (15), we determined the value of the sample's heat conductivity coefficient $\lambda=0.025 \mathrm{~W} \cdot \mathrm{m}^{-1} \cdot \mathrm{K}^{-1} \quad\left(d=0.04 \mathrm{~m}, T_{1}=479 \mathrm{~K}, \quad T_{2}=410 \mathrm{~K}\right.$, $\left.T_{\infty}=297 \mathrm{~K}\right)$.

In comparison to the table heat conductivity of the used material YTONG $\left(\lambda=0.0294 \mathrm{~W} \cdot \mathrm{m}^{-1} \cdot \mathrm{K}^{-1}\right)$, this value of heat conductivity was lower, i.e. the used sample of the foam concrete developed in the Russian Federation showed lower heat conductivity which meant it had a higher heat resistance and thus it was a better insulator.

\section{CONCLUSIONS}

The methods of holographic interferometry open new possibilities for heat transfer but also for the research of physical and mechanical characteristics of strength and deformation of materials [22], [23].

In the contribution we focused on the heat transfer research via natural convection by heating the horizontal sample from the bottom. For the research we used the samples of the new material developed in the Russian Federation - the foam concrete reinforced by PET fibres. The samples were heated by an electric heater from the bottom.

The temperature fields originating above the surface of the horizontal sample were visualised and recorded by the setting of the Mach-Zehnder interferometer onto an infinite width of interference stripes in real time. The quantitative analysis of the holographic interferogram images provided us with the local heat transfer coefficients $\alpha_{\mathrm{x}}$ necessary for subsequent calculation of the heat conductivity coefficient $\lambda$.

Our experiments proved that the more porous the material was, the worse was the material's heat conductivity. The calculated value of the heat conductivity of the newly developed foam concrete $\lambda=0.026 \mathrm{~W} \cdot \mathrm{m}^{-1} . \mathrm{K}^{-1}$ was lower in comparison to the table value of the YTONG material heat conductivity $\left(\lambda=0.0294 \mathrm{~W} \cdot \mathrm{m}^{-1} \cdot \mathrm{K}^{-1}\right)$, i.e. that the used foam concrete sample showed lower heat conductivity, it had higher heat resistance, and as such it was a better insulator.

The boost of ICT together with the development of natural science, technologies, or economics, as well as the development of mathematical analysis provided the science with a massive progress in solving the aforementioned issues. Adequate and efficient computers allow elaborating of a highly accurate analysis of process models describing the physical features of things more precisely [21].

The experiments showed that the used manual regulation of the heat performance is less efficient and that it is very difficult to achieve a stationary process state. Therefore, the electronic regulation of heating with the possibility of setting the required temperature below the tested sample is being prepared. This will help achieve a stable stationary process of heat transfer as well as help gain the possibility of more precise research of thermal-insulation properties of tested materials.

Depending on the purpose and conditions of operation, a new type of reinforced foam concrete can be used as a heat insulating and structural heat insulating material, including in the Far North. It can also be a solution to the problem of sound insulation of compressor stations and busy highways (usage as enclosing panels). This direction requires additional research.

\section{ACKNOWLEDGMENT}

The paper was elaborated with the support of the Ministry of Education, Science, Research and Sports of the Slovak Republic within VEGA 1/0086/18 grant project: Researching temperature fields in a set of shaped heat transfer surfaces, and by the project 013TUKE-4/2019: Modern educational tools and methods for forming creativity and increasing practical skills and habits for graduates of technical university study programmes. 


\section{REFERENCES}

[1] Rabinovich, Ph.N. (2004). Composites Based on Dispersed Reinforced Concrete. Questions of Theory and Design, Technology, Constructions. Moscow, Russia: ASV. (in Russian)

[2] Morgun, V.N., Morgun, L.V., Visnap, A.V. (2015). The usage of reinforcement in fiber reinforced foam concrete products. Construction Materials, 7, 52-54. (in Russian)

[3] Yu, L., Liu, Z. (2016). Mechanical properties optimization of fiber reinforced foam concrete. MATEC $W e b$ of Conferences, 67, 03022. DOI: 10.1051/matecconf/2016703022.

[4] Noor Md. Sadiqul Hasan, Habibur Rahman Sobuz, Md. Shiblee Sayed, Md. Saiful Islam. (2012). The use of coconut fibre in the production of structural lightweight concrete. Journal of Applied Sciences, 12, 831-839. DOI: $10.3923 /$ jas.2012.831.839.

[5] Domnina, K.L., Sotnikova, M.V. (2018). On the issue of the compressive strength of fine-grained fiberreinforced concrete. Science of Udmurtia, 2 (84), 29-33. (in Russian)

[6] Černecký, J., Pivarčiová, E. (2007). Possibilities and Prospects of Holography. Izhevsk, Russia: Izhevsk State Technical University. ISBN 978-5-7526-0303-7.

[7] Mayinger, F., Panknin, W. (1974). Holography in heat and mass transfer. In Proceedings of the 5th International Heat Transfer Conference. Tokyo, Japan: Society of Heat Transfer of Japan, 28-43.

[8] Tauscher, R. (1999). Holographic interferometry in heat and mass transfer. http://citeseerx.ist.psu.edu/ viewdoc/summary?doi=10.1.1.602.7223.

[9] Binu, P.T., Rajendran, K.V., Pillai, S.A. (2006). Wholefield NDT of porous materials using digital holography. In National Seminar on Non-Destructive Evaluation. Indian Society for Non-Destructive Testing, 229-234.

[10] Pavelek, M., Janotková, E., Štětina, J. (2007). Visualisation and Optical Measuremet Methods. Brno, Czech Republic: University of Technolgy in Brno. (in Czech)

[11] Herman, C. (2011). Quantitative visualization of heat transfer in oscillatory and pulsatile flows. In Heat Transfer : Theoretical Analysis, Experimental Investigations and Industrial Systems. IntechOpen, 353-378. DOI: 10.5772/14460.

[12] Lédl, V., Vít, T., Psota, P., Doleček, R. (2011). Holografic interferometry used for measurement of temperature field in fluid. International Journal of Physical and Mathematical Sciences, 5 (11), 17461749. DOI: 10.5281/zenodo.1061908.

[13] Haridas, D., Sobhan, C.B. (2013). Measurement of convective heat transfer from a vertical flat plate using Mach-Zehnder interferometer with wedge fringe setting. International Journal of Mechanical and Mechatronics Engineering, 7 (12), 2385-2390. DOI: 10.5281/zenodo.1089066.
[14] Dančová, P., Vít, T., Lédl, V., Trávníček, Z., Doleček, R. (2013). Holographic interferometry as a tool for visualization of temperature fields in air. Engineering Mechanics, 20 (3/4), 205-212.

[15] Dhote, Y., Thombre, S.B. (2013). A review on natural convection heat transfer through inclined parallel plates. International Journal of Advanced Research in Engineering and Technology, 4 (7), 170-175.

[16] Cernecky, J., Koniar, J., Brodnianska, Z. (2014). The effect of heat transfer area roughness on heat transfer enhancement by forced convection. Journal of Heat Transfer, 136 (4), 041901. DOI: 10.1115/1.4025920.

[17] Verma, S., Joshi, Y.M., Muralidhar, K. (2015). Optical interferometers: Principles and applications in transport phenomena. https://nptel.ac.in/courses/ 112104039/sup_4/article4.pdf.

[18] Zhu, J.R., Cheng, X.M., Dai, J.M., Li, Y.Y. (2015). Temperature measurement of an inclined cylinder using optical interferometry. In International Conference on Artificial Intelligence and Industrial Engineering. Phuket, Thailand, 82-84. DOI: 10.2991/aiie15.2015.23.

[19] Anooplal, B., Binoy, B. (2017). Investigation on heat transfer properties of Nano-fluids using Michelson interferometry. International Advanced Research Journal in Science, Engineering and Technology, 4 (6), 18-22. DOI: 10.17148/IARJSET.

[20] Černecký, J., Koniar, J., Brodnianská, Z. (2011). Optimisation of Heat Exchangers by Using Experimental Methods and Physical Modeling. Zvolen, Slovakia. (in Slovak)

[21] Hart'anský, R., Smieško, V., Rafaj, M. (2017). Modifying and accelerating the method of moment's calculation. Computing and Informatics, 36 (3), 664682. DOI: 10.4149/cai 20173664.

[22] Stejskal, T., Kelemenová, T., Dovica, M., Demeč, P., Stofa, M. (2016). Information contents of a signal at repeated positioning measurements of the coordinate measuring machine (CMM) by laser interferometer. Measurement Science Review, 16 (5), 273-279. DOI: 10.1515/msr-2016-0034.

[23] Sysoev, E., Kosolobov, S., Kulikov, R., Latyshev, A., Sitnikov, S., Vykhristyuk, I. (2017). Interferometric surface relief measurements with subnano/picometer height resolution. Measurement Science Review, 17 (5), 213-218. DOI: 10.1515/msr-2017-0025.

Received March 22, 2019 Accepted July 31, 2019 